\title{
Antimycobacterial activity of five efflux pump inhibitors against Mycobacterium tuberculosis clinical isolates
}

\author{
Guilian $\mathrm{Li}^{1,5}$, Jingrui Zhang ${ }^{1,2,3,5}$, Chao $\mathrm{Li}^{1,3,5}$, Qian Guo ${ }^{1,4}$, Yi Jiang ${ }^{1}$, Jianhao Wei ${ }^{1,3}$, Yan Qiu ${ }^{1}$, Xiuqin Zhao ${ }^{1}$, \\ Li-li Zhao ${ }^{1}$, Jianxin $\mathrm{Lu}^{3}$ and Kanglin Wan ${ }^{1,3}$
}

The Journal of Antibiotics (2016) 69, 173-175; doi:10.1038/ja.2015.101; published online 14 October 2015

Tuberculosis (TB) remains a serious public health threat around the world, with 9 million incident cases and 1.5 million deaths in $2013 .^{1}$ The emergence of multidrug- and extensively drug-resistant TB has made treatment more difficult. Mutations in specific loci of genes associated with an anti-TB drug's mode of action have been considered the main mechanism for drug resistance in Mycobacterium tuberculosis; the active efflux of anti-TB drugs by efflux pumps is an alternative drug resistance mechanism. ${ }^{2}$ Efflux pumps are plasma membrane proteins that have the ability to extrude structurally and functionally unrelated compounds from the cell. The upregulation of efflux systems can significantly decrease the intracellular concentration of many antibiotics, reducing their clinical efficacy. ${ }^{2,3}$

Efflux pump inhibitors (EPIs) have been reported to inhibit the activity of some M. tuberculosis efflux pumps, both in vitro and ex vivo. ${ }^{3-8}$ Therefore, the identification and development of EPIs that can restore the antimicrobial activity of an antibiotic subject to efflux is an approach that merits exploring to prevent the emergence of drug resistance in M. tuberculosis and to improve the efficacy of anti-TB drug treatments. To date, EPIs include phenothiazine drugs such as chlorpromazine (CPZ) and its derivative thioridazine (TZ), carbonyl cyanide m-chlorophenyl hydrazone (CCCP), reserpine (RSP) and verapamil (VP)..$^{3-8}$ CCCP, a proton motive force uncoupler, can reduce the activity of an active efflux system by altering the proton concentration gradient, thereby affecting drug accumulation in the bacteria and restoring drug susceptibility. VP is a calcium channel blocker of ATP-binding cassette transporters. RSP was developed as an antihypertensive drug, but it can also be used as a bacterial efflux inhibitor. ${ }^{7}$ RSP causes bacteria to reduce drug excretion and restores drug sensitivity by reducing the energy supply through ATP hydrolysis. ${ }^{9}$ Phenothiazines drugs have also been shown to inhibit bacterial efflux pumps specifically. ${ }^{10}$ Finally, $\mathrm{TZ}$ is equivalent to $\mathrm{CPZ}$ in all its antimycobacterial properties. ${ }^{11}$

As an alternative drug resistance mechanism in $M$. tuberculosis, efflux pumps have been studied intensely. EPIs were used widely in studies aimed to analyze the role of efflux pumps in drug resistance. MIC tests are usually performed by researchers before choosing a critical concentration of EPI with which to test its effect on modulating the MIC of target drugs in a small number of strains. ${ }^{4-7}$ However, as yet, no reports have been published on the testing of the antimycobacterial activities of EPIs on a large sample of $M$. tuberculosis strains. The aim of this study, therefore, was to determine the antimycobacterial activity of five EPIs (that is, VP, RSP, CPZ, TZ and CCCP) against 92 clinical isolates of $M$. tuberculosis and to evaluate the associations between the MICs and the resistance patterns or genotypes in these isolates. This is the first report of the MIC values of EPIs tested on large-sample-size $M$. tuberculosis strains.

This study obtained approval from the Ethics Committee of the National Institute for Communicable Disease Control and Prevention, Chinese Center for Disease Control and Prevention. The patients with TB included in this research were given a subject information sheet, and they all gave written informed consent to participate in the study.

The 92 M. tuberculosis isolates were obtained from 92 adult patients with pulmonary TB from 2005 to 2010 from six provincial TB hospitals in China, including hospitals in Fujian, Guizhou, Liaoning, Shanxi, Shanghai and Tibet. No epidemiological connection between these patients was detected by standard investigations. Drug susceptibility testing was performed using the Löwenstein-Jensen proportion method (PM) and the critical concentration for the PM was that described previously. ${ }^{12}$ The 92 clinical isolates included 39 isoniazid mono-resistant (INH-MR) isolates, 20 rifampicin mono-resistant (RIF-MR) isolates, 13 multidrug-resistant (MDR) isolates and 20

${ }^{1}$ State Key Laboratory for Infectious Disease Prevention and Control, Collaborative Innovation Center for Diagnosis and Treatment of Infectious Diseases, National Institute for Communicable Disease Control and Prevention, Chinese Center for Disease Control and Prevention, Beijing, China; ${ }^{2}$ Laboratory, Shijiazhuang Obstetrics and Gynecology Hospital, the Fourth Hospital of Shijiazhuang, Shijiazhuang, China; ${ }^{3}$ Key Laboratory of Laboratory Medicine, Wenzhou Medical College, Wenzhou, China and ${ }^{4}$ Pathogenic Biology Institute, University of South China, Hengyang, China

${ }^{5}$ These authors contributed equally to this work.

Correspondence: Professor K Wan, State Key Laboratory for Infectious Disease Prevention and Control, National Institute for Communicable Disease Control and Prevention, Chinese Center for Disease Control and Prevention, P.O. Box 5, Changping, Beijing 102206, China.

E-mail: wankanglin@icdc.cn

or Professor J Lu, Key Laboratory of Laboratory Medicine, Wenzhou Medical College, Wenzhou, Zhejiang 325035, China.

E-mail: jxlu313@163.com

Received 2 April 2015; revised 20 August 2015; accepted 7 September 2015; published online 14 October 2015 
pan-susceptible isolates. The M. tuberculosis standard laboratory strain $\mathrm{H} 37 \mathrm{Rv}$ was included as a control.

In this study, Middlebrook 7H9 broth and ADC supplement were purchased from Difco (Detroit, MI, USA). Alamar blue was purchased from AbD Serotec (Oxford, UK). CCCP, VP, TZ, CPZ and RSP were obtained from Sigma-Aldrich (St Louis, MO, USA).

To determine the MICs of the five EPIs against M. tuberculosis, a 7H9 broth-based microplate Alamar blue assay (MABA) was performed as described previously. ${ }^{13}$ Use of the broth-based methods recommended by the Clinical and Laboratory Standards Institute should decrease the turn-around time and improve international standardization. ${ }^{14}$ MABA based on broth is an inexpensive and reliable method for drug susceptibility testing of $M$. tuberculosis. In this study, all tests for each strain were carried out at least in duplicate to calculate a mean MIC. The final drug concentration ranges were as follows: VP, TZ, CPZ and RSP, 0.5-256.0 $\mu \mathrm{g} \mathrm{ml}^{-1}$; CCCP, 0.1-51.2 $\mu \mathrm{g} \mathrm{ml}^{-1}$. RSP and CCCP were dissolved in dimethyl formamide and dimethyl sulfoxide, respectively. $\mathrm{VP}, \mathrm{TZ}$ and $\mathrm{CPZ}$ were dissolved in sterilized water.

The 92 clinical isolates were genotyped by spoligotyping as previously described by Kamerbeek et al., ${ }^{15}$ and the spoligotyped families were assigned according to the international SpolDB4 database (http://www.pasteur-guadeloupe.fr:8081/SITVITDemo/index.jsp). All Beijing family strains identified by spoligotyping were further grouped into the classical or modern sublineage by PCR to detect the presence or absence of IS6110 in the NTF region. ${ }^{16}$
SPSS 14.0 (SPSS, Chicago, IL, USA) was used to perform the Mann-Whitney and Kruskal-Wallis tests. Before performing these tests, all MIC values were subject to $\log _{2}$ transformation. ${ }^{17}$ The difference was considered to be statistically significant when $P<0.05$.

In the present study, the median and geometric mean MIC values for the $92 \mathrm{M}$. tuberculosis clinical isolates were as follows: 16 and $14.6 \mu \mathrm{g} \mathrm{ml}^{-1}$ for RSP, 3.2 and $4.0 \mu \mathrm{g} \mathrm{ml}^{-1}$ for CCCP, 128 and $123.3 \mu \mathrm{g} \mathrm{ml}^{-1}$ for $\mathrm{VP}, 4$ and $5.5 \mu \mathrm{g} \mathrm{ml}^{-1}$ for $\mathrm{TZ}$, and 8 and $9.0 \mu \mathrm{g} \mathrm{ml}^{-1}$ for $\mathrm{CPZ}$, respectively. The MIC values for $\mathrm{H} 37 \mathrm{Rv}$ were as follows: $32 \mu \mathrm{g} \mathrm{ml}^{-1}$ for RSP, $6.4 \mu \mathrm{g} \mathrm{ml}^{-1}$ for CCCP, $128 \mu \mathrm{g} \mathrm{ml}^{-1}$ for VP, $8 \mu \mathrm{g} \mathrm{ml}^{-1}$ for TZ and $8 \mu \mathrm{g} \mathrm{ml}^{-1}$ for CPZ. The MICs of the five EPIs were distributed in the following ranges: $4-128 \mu \mathrm{g} \mathrm{ml}^{-1}$ for RSP, $1.6-51.2 \mu \mathrm{g} \mathrm{ml}^{-1}$ for CCCP, 32 to $>256 \mu \mathrm{g} \mathrm{ml}^{-1}$ for VP, $2-16 \mu \mathrm{g} \mathrm{ml}^{-1}$ for $\mathrm{TZ}$ and $2-32 \mu \mathrm{g} \mathrm{ml}^{-1}$ for CPZ. These results show that the MIC values of $\mathrm{VP}, \mathrm{TZ}$ and $\mathrm{CPZ}$ varied across a narrow range, whereas the MIC values of RSP and CCCP varied across a wide range. After carefully checking the MIC results, we found that only one of the $92 \mathrm{M}$. tuberculosis isolates had an RSP MIC of $>64 \mu \mathrm{g} \mathrm{ml}^{-1}$, whereas five of the 92 isolates had CCCP MIC values of $>6.4 \mu \mathrm{g} \mathrm{ml}^{-1}$; therefore, we speculate that these clinical isolates are RSP and CCCP resistant. In two previous studies, RSP concentrations of $12 \mu \mathrm{g} \mathrm{ml}^{-1}$, VP concentration of 5 and $40 \mu \mathrm{g} \mathrm{ml}^{-1}$ and CCCP concentrations of $0.5 \mu \mathrm{g} \mathrm{ml}^{-1}, 0.82 \mu \mathrm{g} \mathrm{ml}^{-1}(4 \mu \mathrm{M})$ and $15 \mu \mathrm{g} \mathrm{ml}^{-1}$ were used for all the strains that were tested. ${ }^{3,8,18}$ If bactericidal concentrations of EPIs were used, the activity of other antibiotics would be overestimated, or other erroneous results would be obtained as the M. tuberculosis strains would be killed by the EPIs. Hence, we suggest that sub-inhibition

Table 1 Distribution of MICs ( $\mu \mathrm{g} \mathrm{ml}^{-1}$ ) of five efflux pump inhibitors against Mycobacterium tuberculosis strains

\begin{tabular}{|c|c|c|c|c|c|c|c|c|c|c|c|c|c|c|c|}
\hline \multirow{3}{*}{$\begin{array}{l}\text { Strain type } \\
\text { (number) }\end{array}$} & \multicolumn{3}{|c|}{$R S P^{a}$} & \multicolumn{3}{|c|}{ СССР } & \multicolumn{3}{|c|}{$V P^{a, b}$} & \multicolumn{3}{|c|}{$T Z$} & \multicolumn{3}{|c|}{$C P Z^{a}$} \\
\hline & \multirow[b]{2}{*}{ Median } & \multirow[b]{2}{*}{ Range } & \multicolumn{2}{|c|}{ Geometric } & \multirow[b]{2}{*}{ Range } & \multirow{2}{*}{$\begin{array}{c}\text { Geometric } \\
\text { mean }\end{array}$} & \multirow{2}{*}{ Median } & \multirow[b]{2}{*}{ Range } & \multirow{2}{*}{$\begin{array}{c}\text { Geometric } \\
\text { mean }\end{array}$} & \multirow{2}{*}{\multicolumn{2}{|c|}{ Median Range }} & \multirow{2}{*}{$\begin{array}{c}\text { Geometric } \\
\text { mean }\end{array}$} & \multirow{2}{*}{\multicolumn{2}{|c|}{ Median Range }} & \multirow{2}{*}{$\begin{array}{c}\text { Geometric } \\
\text { mean }\end{array}$} \\
\hline & & & mean & Median & & & & & & & & & & & \\
\hline H37Rv & 32 & - & - & 6.4 & - & - & 128 & - & - & 8 & - & - & 8 & - & - \\
\hline \multicolumn{16}{|l|}{ Clinical isolate (92) } \\
\hline \multicolumn{16}{|c|}{ Resistance phenotype (92) } \\
\hline INH-MR (39) & 16 & $4-128$ & 16.6 & 6.4 & $1.6-51.2$ & 4.8 & 128 & 64 to $>256$ & 150.2 & 4 & $2-16$ & 6.1 & 8 & $2-32$ & 9.7 \\
\hline RIF-MR (20) & 8 & $4-32$ & 11.7 & 3.2 & $1.6-12.8$ & 3.2 & 128 & 64-256 & 97.0 & 4 & $4-8$ & 4.8 & 8 & $4-16$ & 8.3 \\
\hline MDR (13) & 16 & $4-64$ & 14.4 & 3.2 & $1.6-6.4$ & 3.4 & 64 & 32 to $>256$ & 98.0 & 4 & $2-8$ & 4.7 & 8 & $4-16$ & 8.0 \\
\hline Pan-susceptible (20) & 32 & $4-64$ & 14.4 & 3.2 & $1.6-6.4$ & 3.9 & 128 & 64 to $>256$ & 123.6 & 4 & $2-8$ & 5.7 & 8 & $4-16$ & 9.2 \\
\hline \multicolumn{16}{|l|}{ Genotype (92) } \\
\hline Beijing family (52) & 16 & $4-128$ & 12.9 & 3.2 & $1.6-51.2$ & 3.8 & 128 & 64 to $>256$ & 112 & 4 & $2-16$ & 5.3 & 8 & $4-16$ & 8.3 \\
\hline Classical (19) & 8 & $4-64$ & 11.5 & 3.2 & $1.6-51.2$ & 3.7 & 64 & 64 to $>256$ & 106.7 & 4 & $2-16$ & 5.0 & 8 & $4-16$ & 8 \\
\hline Modern (33) & 16 & $4-128$ & 13.8 & 3.2 & $1.6-12.8$ & 3.9 & 128 & 64 to $>256$ & 115.2 & 4 & $4-16$ & 5.5 & 8 & $4-16$ & 8.5 \\
\hline $\begin{array}{l}\text { Non-Beijing family } \\
(40)\end{array}$ & 16 & $4-64$ & 17.1 & 3.2 & $1.6-25.6$ & 4.3 & 128 & 32 to $>256$ & 139.6 & 4 & $2-16$ & 5.8 & 8 & $2-32$ & 10.0 \\
\hline T (15) & 16 & $8-32$ & 17.5 & 3.2 & $1.6-6.4$ & 3.4 & 128 & 64 to $>256$ & 140.4 & 4 & $4-16$ & 5.5 & 8 & $8-32$ & 10.1 \\
\hline U (7) & 16 & 8-32 & 16.0 & 3.2 & $1.6-12.8$ & 4.3 & 128 & $\begin{array}{l}128 \text { to } \\
>256\end{array}$ & 172.3 & 4 & $4-16$ & 5.9 & 16 & $8-32$ & 13.1 \\
\hline$H(5)$ & 16 & $4-32$ & 12.1 & 3.2 & $1.6-6.4$ & 3.7 & 128 & $32-256$ & 97.0 & 4 & $2-16$ & 4.0 & 8 & $2-16$ & 6.1 \\
\hline Orphan (13) & 16 & $8-64$ & 19.8 & 6.4 & $3.2-25.6$ & 6.1 & 128 & 64 to $>256$ & 142.4 & 8 & $4-16$ & 6.8 & 8 & $8-32$ & 10.4 \\
\hline Total (93) & 16 & $4-128$ & 14.6 & 3.2 & $1.6-51.2$ & 4.0 & 128 & 32 to $>256$ & 123.3 & 4 & $2-16$ & 5.5 & 8 & $2-32$ & 9.0 \\
\hline
\end{tabular}

Abbreviations: CCCP, carbonyl cyanide m-chlorophenyl-hydrazone; CPZ, chlorpromazine; INH-MR, isoniazid mono-resistant; MDR: multidrug-resistant; RIF-MR, rifampicin mono-resistant; RSP, reserpine; TZ, thioridazine; VP, verapamil.

${ }^{a}$ The results indicated that the geometric means of the MICs of VP, RSP and CPZ of the Beijing family were significantly lower than that of the non-Beijing family M. tuberculosis isolates

$(P=0.039,0.026$ and 0.049 , respectively).

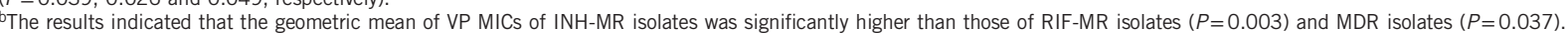

Mann-Whitney and Kruskal-Wallis tests were used to compare the differences between groups with various resistance phenotypes and genotypes. Before performing these tests, all MIC values required $\log _{2}$ transformation. 
concentrations (1/4 MIC or $1 / 2 \mathrm{MIC}$ ) of EPIs should be used in synergy studies.

It has been observed that two-fold variations in MIC values were common when broth microdilution methods are used. ${ }^{19,20}$ Therefore, only comparing the median MIC values between two groups is likely to result in wrong conclusions. Therefore, statistical analysis of a large sample number should reduce the bias produced by the methods. To determine the MIC difference between $M$. tuberculosis groups that carry different-resistant phenotypes, we performed Mann-Whitney and Kruskal-Wallis tests. There was no significant difference in the MIC distributions of RSP, CPZ, TZ and CCCP among the INH-MR, RIF-MR, MDR and pan-susceptible isolates. The median value of the VP MIC values for the INH-MR isolates was identical to that of the RIF-MR isolates, but the geometric mean of the former was significantly higher than that of the latter $(P=0.003)$. We also found that the geometric mean MIC of VP for the INH-MR isolates was significantly higher than that for the MDR isolates $(P=0.037)$. The results suggest that the sensitivity of VP against $M$. tuberculosis clinical isolates depends on the resistance phenotype of this bacterium. However, no significant differences were observed in the MIC distribution of VP between INH-MR and pan-susceptible isolates, between RIF-MR and pan-susceptible isolates, between RIF-MR and MDR isolates or between MDR and pan-susceptible isolates (Table 1).

In $M$. tuberculosis, different genotypes have been reported to be associated with drug resistance. The spoligotyping results for the 92 isolates we tested showed that 52 of them $(56.5 \%)$ belonged to the Beijing family, whereas 40 (43.5\%) belonged to non-Beijing families, the latter which included the $\mathrm{T}$ family (15 isolates), $\mathrm{U}$ family (7 isolates), $\mathrm{H}$ family (5 isolates) and orphans (13 isolates; Table 1). Among the 52 Beijing family strains, $33(63.5 \%)$ were confirmed as modern Beijing strains and $19(36.5 \%)$ were confirmed as classical Beijing strains. Statistical analysis indicated that there was no significant difference in the MIC distributions of TZ and CCCP between Beijing and non-Beijing strains, between modern and classical Beijing strains and among the four lineages of non-Beijing strains. There was no significant difference in the MICs of VP, RSP and CPZ between modern and classical Beijing strains and between the $\mathrm{T}, \mathrm{U}$ and $\mathrm{H}$ family, and orphans of non-Beijing strains. The median values of the MICs of VP, RSP and CPZ of the Beijing family were identical to those of non-Beijing family isolates; however, the geometric means of the MICs of VP, RSP and CPZ of the Beijing family were significantly lower than those of the non-Beijing family M. tuberculosis isolates ( $P=0.039,0.026$ and 0.049 , respectively). These results suggest that the sensitivity of RSP and VP against M. tuberculosis clinical isolates might depend on a specific genotype. More samples should be included to further confirm the MIC distributions of CPZ between Beijing and non-Beijing family strains.

Louw et al. ${ }^{7}$ reported that treatment with VP in combination with first-line drugs could significantly reduce pulmonary colony-forming units in BALB/c mice after 1 and 2 months of treatment. They postulated that EPIs have the potential to improve the efficacy of antiTB drug treatments. ${ }^{7}$ Here, we have reported the median and geometric mean values of the MICs of five EPIs tested on a large sample of $M$. tuberculosis clinical isolates. These results could be referenced in future studies on the treatment of TB.

In conclusion, each of the five EPIs tested herein showed antimycobacterial activity; for VP, CPZ and TZ, the MICs were distributed in a narrow range according to the broth microdilution method. The sensitivity of VP against $M$. tuberculosis clinical isolates may depend on the resistance phenotype and genotype, while the sensitivity of RSP may depend on the genotype of this bacterium. The results of this study suggest that it is important to obtain the MICs of EPIs for individual strains of $M$. tuberculosis and sub-inhibition concentrations (1/4 MIC or $1 / 2 \mathrm{MIC}$ ) of EPIs should be used in synergy studies of EPIs and other antibiotics.

\section{CONFLICT OF INTEREST}

The authors declare no conflict of interest.

\section{ACKNOWLEDGEMENTS}

This work was funded by project 2013ZX10003006-002-001 of the National Key Program of Mega Infectious Disease and the Key Project 2014SKLID104 of State Key Laboratory for Infectious Disease Prevention and Control. The funders had no role in the study design, data collection and analysis, manuscript preparation or decision to publish.

1 World Health Organization. Global Tuberculosis Report 2014. Report No. WHO/HTM/ TB/2014.08 (World Health Organization, Geneva, 2014).

2 De Rossi, E., Ainsa, J. A. \& Riccardi, G. Role of mycobacterial efflux transporters in drug resistance: an unresolved question. FEMS Microbiol. Rev. 30, 36-52 (2006).

3 Pasca, M. R. et al. $\mathrm{mmpL7}$ gene of Mycobacterium tuberculosis is responsible for isoniazid efflux in Mycobacterium smegmatis. Antimicrob. Agents Chemother. 49, 4775-4777 (2005).

4 Rodrigues, L. et al. Contribution of efflux activity to isoniazid resistance in the Mycobacterium tuberculosis complex. Infect. Genet. Evol. 12, 695-700 (2012).

5 Balganesh, M. et al. Rv1218c, an ABC transporter of Mycobacterium tuberculosis with implications in drug discovery. Antimicrob. Agents Chemother. 54, 5167-5172 (2010).

6 Machado, D. et al. Contribution of efflux to the emergence of isoniazid and multidrug resistance in Mycobacterium tuberculosis. PLOS ONE 7, e34538 (2012).

7 Louw, G. E. et al. Rifampicin reduces susceptibility to ofloxacin in rifampicin-resistant Mycobacterium tuberculosis through efflux. Am. J. Respir. Crit. Care Med. 184 269-276 (2011).

8 Gupta, A. K. et al. Microarray analysis of efflux pump genes in multidrug-resistant Mycobacterium tuberculosis during stress induced by common anti-tuberculous drugs. Microb. Drug Resist. 16, 21-28 (2010).

9 Brown, M. H., Paulsen, I. T. \& Skurray, R. A. The multidrug efflux protein NorM is a prototype of a new family of transporters. Mol. Microbiol. 31, 394-395 (1999).

10 Viveiros, M. et al. Isoniazid-induced transient high-level resistance in Mycobacterium tuberculosis. Antimicrob. Agents Chemother. 46, 2804-2810 (2002).

11 Amaral, L., Viveiros, M. \& Kristiansen, J. E. Phenothiazines: potential alternatives for the management of antibiotic resistant infections of tuberculosis and malaria in developing countries. Trop. Med. Int. Health 6, 1016-1022 (2001).

12 World Health Organization. Policy guidance on drug-susceptibility testing (DST) of second-line antituberculosis drugs (WHO/HTM/TB/2008.392) (World Health Organization, Geneva, 2008).

$13 \mathrm{Li}, \mathrm{G}$. et al. Antimicrobial susceptibility of standard strains of nontuberculous mycobacteria by microplate Alamar Blue assay. PLoS ONE 8, e84065 (2013).

14 Clinical And Laboratory Standards Institute. Susceptibility testing of mycobacteria, Nocardiae and other aerobic actinomycetes; approved standard-Second Edition. Document M24-A2,31(5) (Clinical and laboratory standards institute, PA, USA, 2011).

15 Kamerbeek, J. et al. Simultaneous detection and strain differentiation of Mycobacterium tuberculosis for diagnosis and epidemiology. J. Clin. Microbiol. 35, 907-914 (1997).

16 Wada, T., Iwamoto, T. \& Maeda, S. Genetic diversity of the Mycobacterium tuberculosis Beijing family in East Asia revealed through refined population structure analysis. FEMS Microbiol. Lett. 291, 35-43 (2009).

17 Pruller, S. et al. Recommendation for a Standardised Method of Broth Microdilution Susceptibility Testing for Porcine Bordetella bronchiseptica. PLOS ONE 10, e0123883 (2015)

18 Gupta, A. K. et al. jefA (Rv2459), a drug efflux gene in Mycobacterium tuberculosis confers resistance to isoniazid \& ethambutol. Indian J. Med. Res. 132, 176-188 (2010).

19 Jureen, P. et al. Wild-type MIC distributions for aminoglycoside and cyclic polypeptide antibiotics used for treatment of Mycobacterium tuberculosis infections. J. Clin. Microbiol. 48, 1853-1858 (2010).

20 Leonard, B. et al. Inter- and intra-assay reproducibility of microplate Alamar blue assay results for isoniazid, rifampicin, ethambutol, streptomycin, ciprofloxacin, and capreomycin drug susceptibility testing of Mycobacterium tuberculosis. J. Clin. Microbiol. 46, 3526-3529 (2008). 